\title{
INCIDÊNCIA DE CÂNCER GASTROINTESTINAL NO BAIXO AMAZONAS
}

\section{ARTIGO ORIGINAL}

VALENTE, Antonia Regiane Pereira Duarte 1, VALENTE, Gilvandro Ubiracy 2, SANTOS, Larissa Tayná Miranda dos ${ }^{3}$, SILVA, Raissa Carolina Silva e ${ }^{4}$, SILVA, Carla

${ }^{1}$ Mestre em Ciências da Saúde, pós-graduação lato-Sensu em Enfermagem em Unidade de Terapia Intensiva e Metodologias Ativas. Graduada em Bacharel em Enfermagem. ORCID: https://orcid.org/0000-0001-9867-2611

2 Mestre em Ciências da Saúde. Pós-graduação em Gestão de unidade de Saúde, Endoscopia digestiva e Saúde da família. Graduado em medicina pela Universidade Federal do Pará - UFPA. ORCID: https://orcid.org/0000-0003-0781-7024

${ }^{3}$ Graduada em enfermagem pelo Centro Universitário da Amazônia- UNAMA, PósGraduanda em Enfermagem em Ginecologia e Obstetrícia. ORCID: https://orcid.org/0000-0001-5532-2806

${ }^{4}$ Enfermeira Bacharel graduada pelo Centro Universitário da Amazônia - UNAMA, Pós-graduanda em Saúde Pública e PSF. ORCID: https://orcid.org/0000-0002-99408959

RC: 98204

Disponível em: https://www.nucleodoconhecimento.com.br/saude/cancer-gastrointestinal 
Sousa da ${ }^{5}$, SANTOS, Kerolaine Alexsandra Soares dos ${ }^{6}$, SANTOS, Luan Gomes dos 7

VALENTE, Antonia Regiane Pereira Duarte. Et al. Incidência de câncer gastrointestinal no Baixo Amazonas. Revista Científica Multidisciplinar Núcleo do Conhecimento. Ano. 06, Ed. 10, Vol. 01, pp. 05-19. Outubro 2021. ISSN: 2448-0959, Link de acesso: https://www.nucleodoconhecimento.com.br/saude/cancergastrointestinal

\section{RESUMO}

A região do Baixo Amazonas encontra-se localizada estado do Pará, composta por 14 municípios, os quais estão distribuídos em uma extensão territorial de 315 mil km², correspondendo cerca de $9 \%$ da população do referido estado. Considerando que, a referida região possui um quantitativo populacional relevante e, que o câncer tem um histórico epidemiológico de alta incidência mundial, questionou-se: como o câncer gastrointestinal apresenta-se na região do Baixo Amazonas? Trata-se de um estudo com abordagem quantitativa com técnica analítica e descritiva. Tendo como objetivo

${ }^{5}$ Mestranda do Programa de Pós-Graduação em Ciência da Saúde (PPGCSA) do Instituto de Saúde Coletiva (ISCO) da Universidade Federal do Oeste do Pará (UFOPA). Pós-graduanda em Enfermagem em Oncologia e Enfermagem em Unidade de Terapia Intensiva pelo Grupo Educacional IBRA. Bacharel em Enfermagem pelo Centro Universitário da Amazônia - UNAMA. ORCID: https://orcid.org/0000-00025560-8318

${ }^{6}$ Enfermeira, pós graduanda em Enfermagem em Oncologia na Faculdade IBRA, Pós graduanda em enfermagem em Nefrologia pela faculdade UNYLEYA, graduada em Enfermagem pelo Centro Universitário da Amazônia- UNAMA. ORCID: https://orcid.org/0000-0002-7919-1318

7 Pós-graduação em terapia intensiva adulto, Bacharel em Enfermagem. ORCID: https://orcid.org/0000-0002-5730-7578

RC: 98204

Disponível em: https://www.nucleodoconhecimento.com.br/saude/cancer-gastrointestinal 
conhecer a incidência do câncer gastrointestinal no Baixo Amazonas. O mesmo, ocorreu no período de janeiro de 2017 a de junho de 2020. A coleta de dados deu-se a partir dos prontuários dos usuários e arquivos digital disponibilizados por um consultório particular no interior da Amazônia brasileira. Neste cenário, constatou-se que o câncer gastrointestinal se mostra mais prevalente entre os pacientes do sexo masculino, na faixa etária de 61 a 80 anos. Evidencia-se ainda que o segmento mais acometido foi o estômago e o método de elucidação diagnóstica mais utilizado foi a endoscopia digestiva alta e a colonoscopia. Destarte, conclui-se que o câncer é um evento presente e crescente no baixo amazonas, o que pode ser caracterizado como uma situação preocupante para gestores e profissionais de saúde, visto que a assistência relacionada a esse evento é considerada desafiadora e onerosa aos cofres públicos. Assim, sugere-se que novas pesquisas nesse sentido sejam realizadas, buscando por evidências relacionadas ao atendimento nos serviços públicos, uma vez que tais serviços disponibilizam atendimento para todas as classes sociais.

Palavras-chave: Epidemiologia, Oncologia, Diagnóstico.

\section{INTRODUÇÃO}

O câncer é descrito como o crescimento desordenado de células, que se apossa de tecidos e órgãos, de curso e crescimento repentino, as células tendem a ser muito violentas e incontroláveis, instituindo a formação de tumores, capazes de e espalharse para outras regiões do copo, diferenciando-se conforme o tipo de células do corpo acometidas. Nas regiões de tecido epitelial se dão os denominados carcinomas, bem como os de tecido conjuntivo são chamados de sarcomas (INCA, 2018).

Tal eventoe consolidou como uma adversidade, sendo indiscutível as consequências negativas que o crescimento acentuado da sua incidência traz, os quais contribuem diretamente em crises para os sistemas de saúde de diversos países (FRUCHTENICHT et al., 2018).

RC: 98204

Disponível em: https://www.nucleodoconhecimento.com.br/saude/cancer-gastrointestinal 
Santos et al. (2018), enfatizam, que dentre os cânceres que acometem o trato gastrointestinal, os de cólon, reto, estômago, cavidade oral e esôfago ocorrem com maior frequência, em contrapartida as neoplasias de menor frequência são as vesículas biliares, fígado, pâncreas e duodeno. Os autores reiteram que o câncer de cavidade oral, câncer esofágico atingem principalmente pessoas do sexo masculino, e está relacionado principalmente ao tabagismo e etilismo, e baixo nível socioeconômico, os quais são considerados fatores de risco significantes, tanto para o desenvolvimento da patologia, quanto para seu prognóstico.

Na concepção de Carrillo et al. (2018), o câncer gástrico é uma patologia que está ligada diretamente a segunda causa de morte por câncer no mundo e a quinta em incidência anual proveniente de diagnósticos de tumores

Cialkowska-rysz e Zasowska-nowak (2018), enfatizam que o câncer gástrico se apresenta, atualmente, como um dos tumores mais comuns, mantendo-se em alguns países como a neoplasia de maior proporção, além de estar entre os de maior índice de mortalidade. Os autores reiteram ainda que, o tumor gástrico mais comum é o adenocarcinoma, correspondendo a aproximadamente a $95 \%$ de todos os carcinomas gástricos, dos quais parecem estar relacionados a fatores diferentes, como dieta, gastrite atrófica associada ao Helicobacter pylori e anemia perniciosa.

Ma et al. (2018), aditam que as taxas de incidência de câncer de esôfago, estômago e colo retal são de 3,12 e 1,5 casos por 100.00 habitantes. Dos quais o câncer gástrico possui a segunda maior causa de mortalidade, seguido do colo retal, que tem sido o terceiro tumor maligno, mais fatal do mundo.

O câncer gastrointestinal mais frequente é o colorretal, sendo descrito como de causa multifatorial, especificamente as alterações genéticas, exposição ambiental, dieta e condições inflamatórias do trato digestivo. Nos últimos 20 anos obteve a minimização em sua incidência e mortalidade, todavia, os dados corroboram e justificam que é a terceira causa de câncer mais comum quando se relaciona a mortalidade, sendo que

RC: 98204

Disponível em: https://www.nucleodoconhecimento.com.br/saude/cancer-gastrointestinal 
no Brasil, 32.600 novos casos foram diagnosticados em 2013, acarretando 15.070 em homens e 17.530 em mulheres NAHAS et al., 2015).

As ingestas alimentares, exercem interferências de forma direta na carcinogênese gastrointestinais, a qual desempenham tamanha relevância nos estágios iniciais, desse modo, permanece intimamente relacionado com o desenvolvimento do câncer. É possível supor que em um terço de todos os casos de câncer, os fatores nutricionais, bem como o estilo de vida sejam determinantes para o seu surgimento (ALMEIDA et al., 2017).

Alimentações rica em vegetais, frutas, cereais e fibras, além de estilo de vida saudável parece exercer efeito protetor ao câncer do trato gastrointestinal, onde a dieta é apontada em vários estudos como sendo um fator exógeno bastante relevante, chamando atenção para o perigo nas alimentações com altas taxas de cloreto de sódio nitrito e nitrato, existentes em alimentos defumados e frituras (SANTOS et al. 2018)

Souza e Peixoto (2017), ressaltam em seu estudo a necessidade de políticas que subsidiem tanto a atenção primaria quanto os programas que corroboram com aspectos positivos nos determinantes de saúde, visto que se essa base estiver fortalecida consequentemente as internações hospitalares serão minimizadas devido a diminuição do adoecimento.

\section{MATERIAL E MÉTODOS}

Trata-se de um estudo com finalidade fundamental, pois, visa a obtenção de novos conhecimentos. Sendo classificada como uma pesquisa de abordagem quantitativa com técnica analítica e descritiva, visando descrever as características de um determinado fenômeno, utilizando técnicas de padronização de coleta de dados, também se mostra um estudo de caráter retrospectivo, onde visa-se explorar informações a partir de análises documentais em arquivos médicos.

RC: 98204

Disponível em: https://www.nucleodoconhecimento.com.br/saude/cancer-gastrointestinal 
Teve como local de pesquisa um consultório particular no município de Santarém no oeste do Pará. A referida instituição presta atendimento sob forma clínica e diagnóstica, realizando exames de imagens como endoscopia digestiva alta (esofagogastroduodenoscopia) e endoscopia digestiva baixa (colonoscopia), prestando assistência nas especialidades de cínica geral e gastroenterologia sendo referência em diagnóstico oncológico nesta região desde o período de 19 de dezembro de 1986.

O alvo do estudo foram os prontuários dos clientes atendidos na clínica supracitada, de ambos os sexos, com idade igual ou maior a 18 anos, que tenham utilizados os serviços da mesma e tivessem sido diagnosticados com câncer gastrointestinal no período de janeiro de 2017 a de junho de 2020.

Foram inclusos na pesquisa os prontuários dos pacientes que se submeteram a procedimentos diagnósticos, com idade superior a 18 anos e inferior a 90 e que obtiveram o diagnóstico de lesões pré-malignas e câncer do trato gastrointestinal. Sendo excluídos os prontuários dos clientes que utilizaram os serviços somente para consultas e pareceres, que possuíam idade inferior a 18 anos e superior a 90, aqueles que realizaram exames diagnósticos com resultados negativos para lesões malignas do trato gastrointestinal, e ainda aqueles que se encontraram com registros incompletos e/ou ilegíveis.

A coleta de dados deu-se a partir dos prontuários dos usuários e arquivos digital da clínica em estudo, para tal foi utilizado um instrumento semiestruturado, criado pelos pesquisadores, constituído de questões a respeito da situação clínica e socioeconômica dos usuários, incluindo informações sobre antecedentes familiares, exames complementares realizados, bem como a elucidação diagnóstica dos casos.

Os resultados foram analisados através de estatística descritiva, realizadas por meio de números absolutos e porcentagem,

RC: 98204

Disponível em: https://www.nucleodoconhecimento.com.br/saude/cancer-gastrointestinal 
Ressalta-se ainda que, a pesquisa foi realizada em consonância com a Resolução no 466, de 12 de dezembro de 2012, sendo o mesmo submetido, analisado e aprovado pelo comitê de ética e pesquisa (CEP) do Instituto Campinense de Ensino Superior ICES UNAMA, sob o parecer de número 3.211.807.

\section{RESULTADOS E DISCUSSÃO}

O câncer é uma patologia que acomete cerca de 14,1 milhões de pessoas no mundo todo, com taxa de mortalidade de 8,2 milhões e com previsão de aumento nesses números até 2030, pois presentemente, o câncer é considerado uma das doenças que mais debilita e ocasiona a morte do paciente. Os dados abaixo buscam mostrar como se encontra (mesmo que parcialmente) a realidade local.

Nesta primeira tabela optou-se por demonstrar os quantitativos de atendimentos que foram efetivados nos anos de 2017, 2018, 2019 e meados de junho de 2020, na Gastroclínica de Santarém, local que foi escolhido para se obter os dados do presente estudo.

Tabela 1: Relação atendimentos realizados e amostra do estudo.

\begin{tabular}{|l|l|l|l|}
\hline Período & $\begin{array}{l}\text { Total } \\
\text { atendimentos }\end{array}$ & de & Amostra \\
\hline Jan - Dez 2017 & 677 & 61 & $\%$ \\
\hline Jan - Dez 2018 & 710 & 58 & 27,11 \\
\hline Jan - Dez 2019 & 813 & 67 & 29,77 \\
\hline Jan - Jun 2020 & 372 & 39 & 17,33 \\
\hline
\end{tabular}

Fonte: Autores do estudo, com base nos dados da pesquisa.

Santos et al. (2018) classifica o câncer gastrointestinal como uma patologia de causas multifatoriais e com fatores de risco de origem infecciosa, quando causada pela bactéria Helicobacter pylori, distúrbios ou falhas na alimentação, com dietas ricas em

RC: 98204

Disponível em: https://www.nucleodoconhecimento.com.br/saude/cancer-gastrointestinal 
sal, gorduras saturadas, alimentos conservados de variadas formas, como os defumados e dietas pobre em fontes de vegetais, frutas e cereais. Além da exposição frequente ao tabagismo e etilismo, ser portador de algumas doenças, como a gastrite crônica atrófica, metaplasia intestinal da mucosa gástrica, anemia perniciosa, pólipo adenomatoso do estômago e fatores como a idade avançada, sexo masculino, e o histórico pessoal ou familiar de algumas condições hereditárias.

Desconsiderando os tumores de pele não melanoma, os canceres gastrointestinais em homens é o segundo mais frequente nas regiões Norte e Nordeste, o quarto nas regiões Sul e Centro-Oeste e o quinto na região Sudeste. Para as mulheres é o quarto mais frequente na região Norte, o quinto nas regiões Sul e Nordeste e o sexto nas regiões Sudeste e Centro-Oeste (INCA, 2018). Com base no exposto, buscou-se evidenciar como se encontra a distribuição e classificação do referido evento em pacientes atendidos no serviço privado.

Tabela 2: Incidência de câncer gastrointestinal de acordo com sexo.

\begin{tabular}{|l|l|l|l|l|l|}
\hline Período & Ocorrência & \multicolumn{2}{|l|}{ Masculino } & \multicolumn{2}{l|}{ Feminino } \\
\cline { 3 - 7 } & & n & $\%$ & n & $\%$ \\
\hline Jan - Dez 2017 & 61 & 35 & 57,37 & 26 & 42,62 \\
\hline Jan - Dez 2018 & 58 & 27 & 46,55 & 31 & 53,44 \\
\hline Jan - Dez 2019 & 67 & 35 & 52,23 & 32 & 47,76 \\
\hline Jan - Jun 2020 & 39 & 28 & 71,79 & 11 & 28,20 \\
\hline
\end{tabular}

Fonte: Autores do estudo, com base nos dados da pesquisa.

Em seu estudo, Passarin (2020) vem mostrando que as neoplasias continuam em ascensão no Brasil, com uma incidência de 420 mil novos casos no biênio 2018-2019. Evidenciando ainda que houve pouca mudança quanto a distribuição entre os sexos. Porém, nesse contexto o que chama a tenção é que as neoplasias gastrointestinais se encontram presentes em ambos os sexos.

RC: 98204

Disponível em: https://www.nucleodoconhecimento.com.br/saude/cancer-gastrointestinal 
A pesquisa local demonstra dados similares, visto que o sexo masculino é o mais acometido durante o período estudado, entretanto, o sexo feminino ultrapassou no ano de 2018, e não ficou tão distante nos outros anos, o que corrobora com os estudos ao norte mencionados, os quais relatam que os casos de cânceres estão crescendo notavelmente, sendo o sexo masculino mais incidente, o que pode estar relacionado ao fato de que os homens são menos preocupados com as questões pertinentes a saúde e consequentemente tornam-se mais vulneráveis aos fatores de risco.

Assim como outras patologias, a incidência de câncer gastrointestinal aumenta marcadamente conforme a idade. Estudos evidenciam que o risco individual de câncer é estabelecido próximo ou abaixo dos 40 anos e que o aumento de sua expressão ocorre de forma previsível depois disso. Segundo INCA (2018), a incidência aumenta progressivamente com a idade, com maior destaque para a faixa etária entre 50 e 70 anos. Baseado nesses achados buscou-se correlacionar o acometimento de câncer com a faixa etária, ocupação e estado civil. Tais dados serão apresentados na Tabela 3.

Tabela 3: Distribuição de casos de câncer gastrointestinal de acordo com faixa etária, ocupação e estado civil no período de janeiro de 2017 a junho de 2020.

\begin{tabular}{|l|l|l|}
\hline \multicolumn{1}{|r|}{ Faixa etária } & \multicolumn{1}{|c|}{$\%$} \\
\hline Entre 18 a 40 anos & 27 & 12 \\
\hline Entre 41 a 60 anos & 86 & 38,22 \\
\hline Entre 61 a 80 anos & 112 & 49,77 \\
\hline Ocupação & $\mathbf{n}$ & $\%$ \\
\hline Autônomo & 21 & 9,33 \\
\hline Agricultor & 8 & 3,55 \\
\hline Pescador & 14 & 6,22 \\
\hline Do lar & 23 & 10,22 \\
\hline Professora & 11 & 4,88 \\
\hline Aposentados & 75 & 33,33 \\
\hline
\end{tabular}

RC: 98204

Disponível em: https://www.nucleodoconhecimento.com.br/saude/cancer-gastrointestinal 


\begin{tabular}{|l|l|l|}
\hline Outros & 66 & 29,33 \\
\hline Não identificado & 7 & 3,11 \\
\hline Estado civil & $\mathbf{n}$ & $\%$ \\
\hline Solteiro & 56 & 24,88 \\
\hline Casado & 99 & 44 \\
\hline União Estável & 41 & 18,22 \\
\hline Viúvo & 29 & 12,88 \\
\hline
\end{tabular}

Fonte: Autores do estudo, com base nos dados da pesquisa.

A partir dos dados (Tabela 3), é possível evidenciar que a população idosa foi a mais acometida pelo câncer gastrointestinal. O que vem corroborar com um estudo realizado por Cialkowska-Rysz e Zasowska-nowak (2018), no qual o autor mostra que houve predominância de pacientes na faixa etária a partir de 60 anos, fato que estaria relacionado ao aumento da expectativa de vida e à queda na taxa de natalidade, ou seja, predomínio da população de idosos, o que pode resultar no aumento de doenças crônico-degenerativas, como o câncer.

Segundo Bastos et al. (2018), em 2030, o Brasil terá mais idosos do que crianças e jovens, e a mortalidade por câncer terá crescido em uma proporção de $45 \%$. Tal fato está relacionado a diminuição progressiva na capacidade de regeneração e divisão celular em idosos e muitos apresentam alguma comorbidade, o que pode influenciar diretamente no prognóstico da doença e, assim, levá-los à necessidade de cuidados oncológicos paliativos.

O estudo local, ( Tabela 3), constata que a idade feminina mais acometida está na faixa etária entre 51 a 65 anos, ficando 3 anos seguidos em alta no número de atendimentos, o que nos remete a busca um atendimento qualificado mais prévio, não postergando a procura por um especialista, visto que é o correto a se fazer em caso de suspeita de qualquer tipo de câncer, devido o tratamento ser mais eficaz quando

RC: 98204

Disponível em: https://www.nucleodoconhecimento.com.br/saude/cancer-gastrointestinal 
se tem um diagnóstico no começo da doença, principalmente no câncer gastrointestinal, devido a complexa investigação diagnostica.

Quando se trata da relação câncer e ocupação, Bastos et al. (2018), destacam que muitos trabalhadores podem ser expostos a vários carcinógenos durante suas atividades laborativas. E dependendo da ocupação, a falta ou o uso inadequado dos Equipamentos de Proteção Individual (EPIs) se torna bastante relevante para o desenvolvimento de cânceres, como no caso dos agricultores, que devido a exposição com agrotóxicos, que são considerados agentes cancerígenos, pode-se ocorrer uma absorção do produto pela via oral, se alastrando ao longo de todo o trato gastrointestinal, trazendo agravos para saúde do trabalhador.

Sobre a relação nível socioeconômico e câncer gastrointestinal, Santos et al. (2018), apontam que a patologia acomete tipicamente pessoas com classe social mais baixa, apresentando uma proporção de três vezes mais nesse público em relação às classes com um poder aquisitivo mais compensado.

Acredita-se que tal situação pode estar associado aos fatores de risco, como as falhas na alimentação, onde pessoas com o nível socioeconômico mais baixo não se tem tantas oportunidades para buscar atendimento específico para saúde nutricional e consequentemente acabam usufruindo de uma alimentação não tão saudável, com dietas ricas em sal, gorduras saturadas, alimentos conservados de variadas formas, como os defumados e dietas pobre em fontes de vegetais, frutas e cereais.

O que corrobora com os dados do estudo atual o qual evidencia que a maioria dos pacientes analisados eram aposentados e do lar, dos quais muito já se sabe que a renda não é considerada tão adequada para suas demandas, sendo seguido de outras ocupações como autônomos, agricultores, professoras, pescadores, entre outros.

Quando uma pessoa é diagnosticada com câncer ela necessitada de alguém do seu lado, pois esse é um momento muito difícil, visto que surgem vários receios e 
preocupações. Assim, saber que se tem alguém do lado é de fundamental importância para o psicológico.

Corroborando com os achados locais, Bastos et al. (2018) mostram em sua pesquisa que, 122 encontravam-se casadas/união estável, 62 solteiras, 26 viúvos, 10 divorciados e 19 não deram informação, com um resultado de 51, 05\% observou-se que grande parte dos pacientes se encontravam casados. Neste sentido, Bastos et al (2018), ponderam que não se pode estabelecer relação entre o estado civil e a ocorrência de câncer avançado e sem possibilidades de cura. Na percepção dos autores, tal fato não influencia no prognóstico da doença.

A análise em curso demonstra que a maioria dos pacientes atendidos na clínica encontravam-se casados, podendo correlacionar este fato com a questão de que as pessoas que vivem em um relacionamento acabam se cuidando mais, o casamento pode auxiliar na melhoria da saúde quando os envolvidos copiam os hábitos saudáveis um do outro, incentivam as consultas médicas, podendo descobrir alguma patologia presente e precocemente começar o tratamento, que no caso do câncer, tal situação mostra-se positiva, uma vez que, quanto mais cedo é diagnosticado e tratado, melhores chances de cura.

Em um estudo de Santos et al (2018), é enfatizado que dentre os cânceres que acometem o trato gastrointestinal, os de cólon, reto, estômago, cavidade oral e esôfago ocorrem com maior frequência, em contrapartida as neoplasias de menor frequência são as vesículas biliares, fígado, pâncreas e duodeno.

Tabela 4: Distribuição do câncer gastrointestinal de acordo com os seguimentos acometidos, com método de elucidação diagnostica e com o estágio de Borrmann.

\begin{tabular}{|l|l|l|}
\hline Segmento acometido & $\mathbf{n}$ & $\%$ \\
\hline Boca & 10 & 4,44 \\
\hline Esôfago & 12 & 5,33 \\
\hline
\end{tabular}

RC: 98204

Disponível em: https://www.nucleodoconhecimento.com.br/saude/cancer-gastrointestinal 


\begin{tabular}{|c|c|c|}
\hline Estomago & 121 & 53,77 \\
\hline Cólon & 25 & 11,11 \\
\hline Duodeno & 04 & 1,77 \\
\hline Reto & 17 & 7,55 \\
\hline Pâncreas & 13 & 5,77 \\
\hline Fígado & 09 & 4,00 \\
\hline Método diagnóstico & $\mathbf{n}$ & $\%$ \\
\hline Endoscopia Digestiva alta - EDA & 52 & 23,11 \\
\hline Colonoscopia & 57 & 25,33 \\
\hline Biópsia & 21 & 9,33 \\
\hline Classificação a partir da EDA & $\mathbf{n}$ & $\%$ \\
\hline Borrmann I & 1 & 1,92 \\
\hline Borrmann II & 2 & 3,84 \\
\hline Borrmann III & 11 & 21,15 \\
\hline Borrmann IV & 8 & 15,38 \\
\hline
\end{tabular}

Fonte: Autores do estudo, com base nos dados da pesquisa.

Carrillo et al. (2018), enfatizam que o câncer gástrico se apresenta, atualmente, como um dos tumores mais comuns, mantendo-se em alguns países como a neoplasia de maior proporção, além de estar entre os de maior índice de mortalidade. O tumor gástrico mais comum é o adenocarcinoma, correspondendo a aproximadamente a $95 \%$ de todos os carcinomas gástricos.

Segundo dados do INCA (2018), o câncer de cólon e reto tem forte relação com o estilo de vida do paciente, já que sua incidência se eleva em regiões com hábito alimentar rico em consumo de carne vermelha e processadas, sem nenhum ou mínimo de consumo de verduras, legumes ou frutas. Além do excesso em consumo de álcool e tabaco, levando um padrão de vida sedentário, sem exercer qualquer atividade física, podendo evoluir para a obesidade 
De acordo com Fortes (2015), o câncer de esôfago é o considerado o sexto tipo mais frequente de neoplasia entre os homens e entre as mulheres ocupa a 15a posição, apresentando altos índices de letalidade. E uma grande parte dessas neoplasias são do tipo epidermóides, que se localizam nas porções médias e superiores do esôfago, que possuem forte relações com o tabagismo e o alcoolismo, o que eleva o risco da doença se desenvolver de forma mais rápida. Já o adenocarcinoma que se desenvolve na porção distal do esôfago, tem intima relação com a obesidade, elevando o risco da doença se desenvolver 40 vezes em pessoas com o índice de massa corpórea acima de $30 \mathrm{~kg} / \mathrm{m}$.

Dados esses que colaboram com a pesquisa local, que apontam os canceres de estomago, cólon, reto, esôfago e boca como os mais incidentes entre as neoplasias gastrointestinais, estando associado aos fatores que desenvolvem os mesmos, como o estilo de vida, padrão alimentar, tabagismo, etilismo e a obesidade. Já que na região do baixo amazonas tem um alto índice de consumo de carnes vermelhas, gorduras saturadas, além do público predominante da pesquisa, idade igual ou maior que 51 anos, chegando até os 80 e terem pouca prática de exercícios físico, o que contribui para o desenvolvimento das neoplasias.

Com relação ao aspecto macroscópico, a classificação mais utilizada para o Câncer Gástrico Avançado (CGA) é a de Borrmann, que apresenta maior uniformidade descritiva endoscópica, (LUO et al., 2016).

O método diagnostico endoscópio é realizado a partir de um aparelho fibroendoscopio que permite uma avaliação mais detalhada da cavidade gástrica, avaliando mucosas adjacentes e lesões, obtendo uma amostra para análise histopatológica através de biópsia. Os aspectos macroscópicos comumente descritos são o Ulcerado ou Úlceroinfiltrativo (Borrmann II e III, respectivamente), com comprometimento da distensibilidade e motilidade da parede gástrica ao exame endoscópico.

Segundo Souza et al. (2013), a sensibilidade da biópsia endoscópica para o diagnóstico do CGA permanece elevada, sendo o método diagnóstico de escolha. No RC: 98204

Disponível em: https://www.nucleodoconhecimento.com.br/saude/cancer-gastrointestinal 
estudo em questão, aproximadamente $16 \%$ dos pacientes com CGA tiveram biópsias negativas para malignidade na avaliação endoscópica inicial, a sensibilidade da biópsia endoscópica situa-se entre 90-95\%. Como os achados nas lesões infiltrativas são sutis, um exame minucioso com um aparelho adequado permite maior precisão da identificação de tais lesões. Os endoscópios modernos permitem maior detalhamento na avaliação das lesões gástricas, permitindo notar alterações discretas na parede gástrica.

Com isso obteve-se os resultados que foram demonstrados na Tabela 4, entretanto, não foi possível se obter $100 \%$ dos resultados dos diagnósticos, devido o tempo de pesquisa e o fato que alguns pacientes não retornarem para realização de novos exames quando necessário, assim como também não retornarem com seu histopatológico que é realizado fora da clínica estudada, não havendo como elucidar o diagnóstico.

A classificação de Borrmann é a mais utilizada entre os endoscopistas para classificar o tipo de neoplasia, onde Borrmann I é a Lesão polipóide ou vegetante, bem delimitada. Borrmann II Lesão ulcerada, bem delimitada, de bordas elevadas. Borrmann III Lesão ulcerada, infiltrativa em parte ou em todas as suas bordas e Borrmann IV, definido como de caráter infiltrativo, pode acometer o estômago de forma difusa ou localizada, de acordo com a extensão do envolvimento gástrico.

De acordo com Luo et al. (2016), as formas avançadas são estudadas morfologicamente segundo a classificação de Borrmann e a forma precoce de acordo com a classificação Sociedade Japonesa de Câncer Gástrico. E seu tipo histológico pela classificação de Lauren. Os autores reiteram ainda que a maioria dos pacientes sintomáticos já apresenta uma doença avançada e incurável no momento do diagnóstico. Na concepção destes, lesões precoces são normalmente assintomáticas e não frequentemente detectadas fora do âmbito de screenings. Dentre os sinais e sintomas mais comuns os referidos autores citam a perda de peso e dor abdominal. 
O prognóstico do paciente oncológico está intimamente ligado ao tipo de lesão e o seu grau de estadiamento, pois quanto menor o estadio, maior será as chances de sobrevida desse paciente. Fortes (2015), considera que no Brasil, apenas 10\% a 15\% dos casos de neoplasias gástricas, são diagnosticadas na fase inicial, o que leva as taxas de sobrevida dos pacientes serem bem inferiores, não ultrapassando a marca dos $30 \%$.

\section{CONCLUSÃO}

O estudo em pauta possibilitou evidenciar que o câncer é um evento presente e crescente no baixo amazonas, o que pode ser caracterizado como uma situação preocupante para gestores e profissionais de saúde, visto que a assistência relacionada a esse evento é considerada desafiadora e onerosa aos cofres públicos.

\section{REFERÊNCIAS}

ALMEIDA, Liliane; SANTOS, Brenda Taimara; PRATES, Rodrigo pereira. et al. Alimentação como fator de risco para câncer de intestino em universitários. Fortaleza (CE). Revista Brasileira em promoção da Saúde. v. 30, n. 1, p. 72-78, jan./mar., 2017. DOI: 10.5020/18061230.2017.p72.

BASTOS, Bárbara Rafaela; PEREIRA, Adrya Karolinne da Silva; CASTRO, Cinthia Costa de et al. Perfil sociodemográfico dos pacientes em cuidados paliativos em um hospital de referência em oncologia do estado do Pará, Brasil, 2018. Rev Pan-Amaz $\begin{array}{llllll}\text { Saude. v.9, n.2, } & \text { p. } & 31 \quad-\quad 36 . \text { Ananindeua jun. } 2018 . \quad \text { DOI: }\end{array}$ http://dx.doi.org/10.5123/s2176-62232018000200004

CARRILLO, Gloria Mabel; BAYONA, Herman Arnulfo; ARIAS, Edier Mauricio. Perfil y carga de la enfermedad de personas con cáncer gástrico sometidas a gastrectomía. rev.fac.med.v.66, n.1, p $13 \quad-18$, Bogotá Jan./Mar. 2018. DOI: http://dx.doi.org/10.15446/revfacmed.v66n1.60273

RC: 98204

Disponível em: https://www.nucleodoconhecimento.com.br/saude/cancer-gastrointestinal 
CIAŁKOWSKA-RYSZ, Aleksandra; ZASOWSKA-NOWAK, Anna. The diagnosis and treatment in palliative care patients. Medycyna Paliatywna 2018; v.10, n.1, p. 1923 DOI: https://doi.org/10.5114/pm.2018.77202

FRUCHTENICHT, Ana Valéria Gonçalves; POZIOMYCK, Aline Kirjner; REIS, Audrey Machado dos; et al. Estado inflamatório e nutricional em pacientes submetidos à ressecção cirúrgica de tumores do trato gastrointestinal. Revista do Colégio Brasileiro de Cirurgião. Porto Alegre (RS). v. 45, n. 2, p. 1-11, 2018. DOI: 10.1590/0100-6991e-20181614.

INSTITUTO NACIONAL DE CÂNCER JOSÉ ALENCAR GOMES DA SILVA - INCA. ABC do câncer: abordagens básicas para o controle do câncer. Organização Mario Jorge Sobreira da Silva. - 4. ed. rev. atual. - Rio de Janeiro: Inca, 2018. 111 p. ISBN 978-85-7318-351-1 (versão eletrônica). Disponível em: https://www.inca.gov.br/sites/ufu.sti.inca.local/files/media/document/livro-abc-4edicao.pdf. Acesso em: 20 de outubro de 2019.

FORTES, Renata Costa; VIEIRA, Angélica Reis. Qualidade de vida de pacientes com câncer gastrointestinal. Com. Ciências Saúde, v.26, n. 1/2. P.45 -46, 2015. Disponível

em: https://bvsms.saude.gov.br/bvs/periodicos/ccs_artigos/2015_qualidade_vida_pacient es.pdf. Acesso em 21 de nov. 2019.

LUO, Yifan; GAO, Peng; SONG, Yongxi. et al. Clinicopathologic characteristics and prognosis of Borrmann type IV gastric cancer: a meta-analysis . World J Surg Onc. 2016. v.14, n.49, p. 14 - 49. DOI: https://doi.org/10.1186/s12957-016-0805-9

MA, Xueni; GAO, Ya, LI Jipin; et al. The association between body mass index and the risk of different gastrointestinal cancers: A protocol for an overview of systematic reviews. Medicine (Baltimore). 2018. v.97, n.45, p.e13181. doi: 10.1097/MD.0000000000013181. PMID: 30407353; PMCID: PMC6250501.

RC: 98204

Disponível em: https://www.nucleodoconhecimento.com.br/saude/cancer-gastrointestinal 
NAHAS, Sergio Carlos; NAHAS, Caio Sergio Rizkallah , BUSTAMANTE-LOPEZ Leonardo Alfonso; et al. FATORES PROGNÓSTICOS DE PACIENTES COM CÂNCER DE CÓLON DIREITO TRATADOS CIRURGICAMENTE: Experiência de 10 anos de uma instituição universitária. ABCD Arq Bras Cir Dig . v.28, n.1, p.3-7, São Paulo: 2015. DOI:http://dx.doi.org/10.1590/S0102-67202015000100002

PASSARIN, Nayara de Paula; PEREIRA, Antonio Augusto Claudio; COIMBRA, Jordana Henriques; et. al. Avaliação da Qualidade de Vida e Prevalência de Sintomas Depressivos em Pacientes Oncológicos Submetidos à Radioterapia. Revista Brasileira de Cancerologia 2020; v. 66, n.1, p. e-12775. DOI: https://doi.org/10.32635/2176 9745.RBC.2020v66n1.775

SANTOS, Sérgio Sousa Sena; MAGALHÃES, Magnólia de Jesus Sousa; ARAGÃO, Francisca Bruna Arruda; et al. Perfil clínico e epidemiológico de pacientes com câncer gástrico em um hospital de referência. Brazilian Journal of Surgery and Clinical Research - BJSCR. V.23,n.2,p.24-28 (Jun - Ago 2018). ISSN online: 2317-4404. Disponível em: https://www.mastereditora.com.br/periodico/20180704_094357.pdf. Acesso em: 25 de nov. 2019.

SOUZA, Dayane Kelle de; PEIXOTO, Sérgio Viana. Estudo descritivo da evolução dos gastos com internações hospitalares por condições sensíveis à atenção primária no Brasil, 2000-2013. Epidemiol. Serv. Saude, Brasília, v26, n.2, p. 285-294, abr-jun 2017. DOI: 10.5123/S1679-49742017000200006

SOUZA, Benilton Batista de; ARAÚJO, Nayana Camurça de ; YAMANAKA, Ademar. Câncer gástrico avançado: Acurácia da biópsia endoscópica. Rev. Ciênc. Méd. Biol., Salvador, v.12, n.3, p.299-305, set./dez. 2013. ISSN 1677-5090. Disponível em: https://repositorio.ufba.br/ri/bitstream/ri/23091/1/6_v.12_3.pdf. Acesso em: 28 de nov. de 2019.

Enviado: Julho, 2021.

RC: 98204

Disponível em: https://www.nucleodoconhecimento.com.br/saude/cancer-gastrointestinal 
Aprovado: Setembro, 2021.

RC: 98204

Disponível em: https://www.nucleodoconhecimento.com.br/saude/cancer-gastrointestinal 